\title{
Alcohol Intake and Risk of Ischemic and Haemorrhagic Stroke: Results from a Mendelian Randomisation Study
}

\author{
Anne I. Christensen, ${ }^{a}$ Børge G. Nordestgaard, ${ }^{\mathrm{b}, \mathrm{c}, \mathrm{d}}$ Janne S. Tolstrup ${ }^{\mathrm{a}}$ \\ ${ }^{a}$ National Institute of Public Health, University of Southern Denmark, Copenhagen, Denmark \\ ${ }^{b}$ Department of Clinical Biochemistry, Herlev and Gentofte Hospital, Copenhagen University Hospital, Herlev, Denmark \\ 'The Copenhagen General Population Study, Herlev and Gentofte Hospital, Copenhagen University Hospital, Herlev, Denmark \\ 'The Copenhagen City Heart Study, Frederiksberg Hospital, Copenhagen University Hospital, Frederiksberg, Denmark
}

Background and Purpose To test whether alcohol intake, both observational and estimated by genetic instruments, is associated with risk of ischemic and haemorrhagic stroke.

Methods We used data from the Copenhagen City Heart Study 1991 to 1994 and 2001 to 2003, and the Copenhagen General Population Study 2003 to 2012 ( $n=78,546)$. As measure of alcohol exposure, self-reported consumption and genetic variation in alcohol metabolizing genes (alcohol dehydrogenase $A D H 1 B$ and $A D H 1 C$ ) as instrumental variables were used. Stroke diagnoses were obtained from a validated hospital register.

Results During follow-up 2,535 cases of ischemic and haemorrhagic stroke occurred. Low and moderate alcohol intake ( 1 to 20 drinks/week) was associated with reduced risk of stroke. The hazard ratios associated with drinking 1 to 6,7 to 13 , and 14 to 20 drinks/week were 0.84 (95\% confidence interval [Cl], 0.76 to 0.92$), 0.83(95 \% \mathrm{Cl}, 0.73$ to 0.94$)$, and $0.84(95 \% \mathrm{Cl}, 0.73$ to 0.97$)$, respectively, compared with drinking $<1$ drink/day. $A D H 1 B$ and $A D H 1 C$ genotypes were not associated with risk of stroke. Further analysis to test the included measures revealed that increasing alcohol intake (per 1 drink/day) was positively associated with risk of alcoholic liver cirrhosis, but not associated with risk of stroke, and that increasing blood pressure (per systolic $10 \mathrm{~mm} \mathrm{Hg}$ ) was not associated with risk of alcoholic liver cirrhosis, but positively associated with risk of stroke.

Conclusions Low and moderate self-reported alcohol intake was associated with reduced risk of stroke. The result was not supported by the result from the causal genetic analysis.

Keywords Alcohol drinking; Incidence; Stroke; Prospective studies; Mendelian randomisation
Correspondence: Janne S. Tolstrup National Institute of Public Health, University of Southern Denmark, Øster Farimagsgade 5A, 2., 1353 Copenhagen, Denmark

Tel: +45-6550-7735

E-mail: jst@niph.dk

Received: July 5, 2017

Revised: November 24, 2017

Accepted: December 24, 2017

\section{Introduction}

Alcohol is consumed widely throughout the world. In Western countries, abstainers constitute the minority while most have a light to moderate alcohol intake (drinking a max of 2 and 3 drinks per day for women and men). Harms associated with this habit are therefore of huge public health and of potential clinical concern.

The association between alcohol intake and stroke risk has been addressed in a number of epidemiological studies, with differing results; especially controversy exists whether drinking in moderation confers a reduced risk of ischemic stroke, in accordance with some studies, ${ }^{1-5}$ and increased risk of haemorrhagic stroke. ${ }^{1,6}$ Moderate alcohol intake was not associated 
with stroke, ischemic or haemorrhagic, in most studies, s.7-11 $^{3}$ which however in a recent meta-analysis was summed up to a significantly reduced pooled relative risk (RR) of ischemic stroke for a low intake ( $<15 \mathrm{~g}$ equalling approximately 1.3 drinks per day) compared with abstaining. ${ }^{12}$ However, especially the largest individual studies that took part of the meta-analysis contributed with insignificant findings with statistical estimates near 1.0, while studies of smaller size had results further away from the null, all in all indicating that a "protective effect" of alcohol on ischemic stroke is either small or that bias is present. Another meta-analysis also concluded that light to moderate drinking ( $\leq 2$ drinks/per day) was associated with a reduced risk of ischemic stroke and heavy drinking ( $>4$ drinks) per day) was associated with increased risk of both ischemic and haemorrhagic stroke. ${ }^{13}$

Generally, when studying health effects associated with drinking in moderation, one has to rely on the participants' own report, implying possibility of reverse causality and confounding. Disentangling causal effects of alcohol from effects of related life style factors is likely to be especially difficult at the low to moderate intake level, as difference in true risk is small compared with the reference group of non-drinkers. Complicating such comparison further is the facts that nondrinkers often constitute a heterogeneous group of individuals who abstain by simple preference and individuals who abstain due to health or socioeconomic reasons. For instance, relatively more abstainers than light drinkers have chronical diseases and low socioeconomic position. ${ }^{14,15}$

An attempt to address the problems associated with self-reported information is to use the Mendelian randomization design, a method that uses genetic variants as instrumental variables for the exposure, instead of the exposure itself. ${ }^{16,17}$ For alcohol, functional variants in genes encoding alcohol dehydrogenase (ADH), responsible for the majority of the body's alcohol degradation, are associated with extent of alcohol intake: individuals who have the fast alcohol degrading alleles $A D H 1 B^{*} 2$ and $A D H 1 C^{*} 1$ consume less alcohol than those with slow degrading alleles $A D H 1 B^{*} 1$ and $A D H 1 C^{*} 2$, which persists across age groups from early adulthood to old age, and observed in various populations. ${ }^{18-22}$ In general, instruments valid for Mendelian randomisation (MR) analysis should be associated with the exposure of interest (in this case, amount of alcohol intake) and should influence the outcome through that exposure only ${ }_{i}^{23}$ i.e., there should be no pleiotropic effects. For the alcohol dehydrogenase $1 \mathrm{~B}$ and $1 \mathrm{C}(\mathrm{ADH} 1 \mathrm{~B}$ and $\mathrm{ADH} 1 \mathrm{C})$ genotypes, no pleiotropic effects have been identified. ${ }^{24}$ All taken together, $A D H$ genotypes are valid candidates for unbiased instruments of lifelong alcohol consumption.
In this study we tested the hypothesis that alcohol intake is associated observationally and causally through genetic variation with risk of any, ischemic and haemorrhagic stroke. For this purpose, we used data from two large population-based cohorts with information on self-reported alcohol consumption and genetic variations in $A D H 1 B$ and $A D H 1 C$ as instrumental variables.

\section{Methods}

\section{Study populations}

The Copenhagen General Population Study (CGPS) is an ongoing prospective study of the Danish general population initiated in 2003. To be eligible, participants had to be of Danish descent (i.e., the participant and both parents were born in Denmark and were Danish citizens). Among individuals living in counties of greater Copenhagen aged 20 to 40 years, 25\% were invited, whereas all eligible people aged $>40$ years were invited. The response rate was $42 \%$. When the present study was initiated, 84,720 individuals had been included.

The Copenhagen City Heart Study (CCHS) was initiated in 1976 where a random sample of the Danish general population above age 20 years living in the Center of Copenhagen was invited to participate (number of participants, 14,223; response rate, 74\%). This examination was followed by three examinations; a second examination in 1981 to 1983, where all previously invited plus 500 new individuals aged 20 to 24 years were invited $(n=12,698,70 \%)$; a third examination in 1991 to 1994 where all previously invited plus 3,000 new individuals aged 20 to 49 years were invited $(n=10,135,61 \%)$; and a fourth examination in 2001 to 2003 where all previously invited plus an additional sample of 1,040 individuals aged 20 to 29 years were invited $(n=6,238,50 \%)$. Eligibility criterion was Danish citizenship, and the participants does not reflect the ethnic admixture of Copenhagen (for instance, the proportion of inhabitants with foreign citizenship was $8 \%$ in 1994). However, even a few participants of foreign ethnicity could confound our results since the $A D H 1 B^{*} 2$ allele has shown considerable population stratification. ${ }^{25}$ Hence, information on birth place as recorded in the Danish Civil Registration System ${ }^{26}$ was obtained, and participants who were born in Asia, Africa, the Middle East, South America, and Greenland were excluded $(n=243)$.

We used data from CCHS 1991 to 1994 and 2001 to 2003 examinations $(n=11,365)$ and the CGPS 2003 to 2012 examination $(n=84,720) .^{27-29}$ Data were collected following similar principles. Participants filled in a questionnaire that was reviewed by an investigator on the day of attendance, had a 
physical examination performed, and had blood samples drawn for biochemical measurements and DNA extraction. Written informed consent was obtained. The Danish Ethics Committee approved the studies (No. 100.2039/91 and H-01-144/01).

\section{Assessment of alcohol consumption}

Participants reported their consumption of beer (in bottles), wine (in glasses), and spirits (in units) with response categories of "never/ hardly ever," "monthly," "weekly," or "daily" and number of drinks per week. The total number of drinks per week was calculated by summing up the individual types of alcohol and was categorised as in previous studies: $:^{30} 1$ to 6,7 to 13,14 to 20,21 to 27,28 to 34 , and $\geq 35$ drinks per week with 1 drink equalling $12 \mathrm{~g}$ alcohol. For women, the highest category was $\geq 28$ drinks per week.

Previously, we have shown that stepwise increments of the self-reported alcohol consumption consistently corresponded to stepwise increments in factors expected to correlate with amount of alcohol consumption (alanine aminotransferase, $\gamma$-glutamyl transpeptidase, erythrocyte volume, and alkaline phosphatase), speaking in favour of the validity of self-reported alcohol consumption. ${ }^{18}$

\section{$A D H 1 B$ and $A D H 1 C$ genotypes}

DNA was isolated from full blood and stored at $-45^{\circ} \mathrm{C}$. Participants were genotyped for the ADH1B genotype (rs1229984; Arg47His) and $A D H 1 C$ genotype (rs698; lle349Val) by Nanogen Technology (Carson City, NV, USA) ) $^{31,32}$ and TaqMan assays (Applied Biosystems, Foster City, CA, USA). ${ }^{19}$ Genotypes were in HardyWeinberg equilibrium in both studies ( $P>0.05$ ). Genotypes $A D$ $H 1 B^{*} 2 / 1$ and $A D H 1 B^{*} 2 / 2$ were grouped because of few participants with these genotype. $A D H 1 B$ and $A D H 1 C$ genotypes were combined and ranked according to expected enzyme activity, ranging from most to least active $\left(1, A D H 1 B^{*} 2 / x+A D H 1 C^{*} 1 / 1 ; 2\right.$, $A D H 1 B^{*} 1 / 1+A D H 1 C^{*} 1 / 1 ; 3, A D H 1 B^{*} 1 / 1+A D H 1 C^{*} 1 / 2 ; 4$, $A D H 1 B^{*} 1 / 1+A D H 1 C^{*} 2 / 2$ ). Additionally, we used regression analysis with alcohol intake as a dependent variable and sex and genotypes as independent variables to test how much of the variance in alcohol consumption was explained by the $A D H 1 B$ and $A D H 1 C$ genotypes.

\section{Assessment of ischemic and haemorrhagic stroke} Information on diagnosis of stroke was obtained from the Danish National Patient Register that holds records of all Danish hospital admissions since 1978 and further outpatient and emergency ward contacts since 1995, and from the Danish Causes of Death Registry which contains information on causes of death for all deceased in Denmark. ${ }^{33-35}$ In both registers, re- cords are classified according to World Health Organization (WHO) International Classification of Diseases (ICD). Records with the following codes implying stroke were extracted: ICD8431 to 438 and ICD-10 I60 to I68 and G45.

All diagnoses of stroke were validated individually. Records were requested from general practitioners and hospitals and validated by two experienced medical doctors independently, blinded to the test results. ${ }^{36,37}$ Ischemic stroke was defined according to WHO criteria as rapidly developing signs of focal or global disturbance of cerebral function lasting $>24$ hours (unless interrupted by death), with no apparent nonvascular cause. ${ }^{38}$ Haemorrhagic stroke and subarachnoidal hemorrhage were excluded from the ischemic stroke group. To distinguish among infarction, intracerebral hemorrhage, and subarachnoidal hemorrhage, either computed tomography or magnetic resonance imaging scan, spinal fluid examination, autopsy, or surgical description were required. If the scan did not show infarction or hemorrhage, but symptoms in accordance with stroke criteria, that event was diagnosed as ischemic stroke. The diagnosis of ischemic and haemorrhagic stroke was not applied in cases in which a scan revealed signs of prior cerebrovascular disease, but without history of any symptoms, if symptoms were nonfocal, or if symptoms lasted $<24$ hours. ${ }^{39}$ Alcoholic liver cirrhosis was ascertained similarly from the Danish registries using ICD-8 code 571.0 and ICD-10 code k70.3.

\section{Statistical analysis}

Of the initial sample of 89,675 individuals, we excluded those with stroke prior to baseline $(n=2,737)$, missing information on alcohol consumption $(n=4,499)$ and covariates (smoking $[n=2,573])$, school education $(n=247)$, body mass index (BMI) $(n=511)$, and leisure time physical activity $(n=562)$. The final analytic sample therefore consisted of 78,546 men and women (where genotype information was available for 74,632 individuals) (Table 1).

Variables considered being potential confounders included the following: age; school education $(<8,8$ to 11 , and $>11$ years of education, corresponding to lower primary school, higher primary school, and secondary school); leisure time physical activity (sedentary, light, moderate, and vigorous); smoking (never smoker, former smoker, and current smoker of 1 to 14,15 to 24 , and $>24 \mathrm{~g}$ of tobacco/day); BMI (linearly); family history of cardiovascular disease (either biological parent having had myocardial infarction or stroke); diabetes (by self-report), angina pectoris (by self-report), hypertension (by self-report); ischemic heart disease (hospital diagnosis before baseline, yes/no); congestive heart failure (hospital diagnosis before baseline, yes/no); angina pectoris (by self-report); heart 


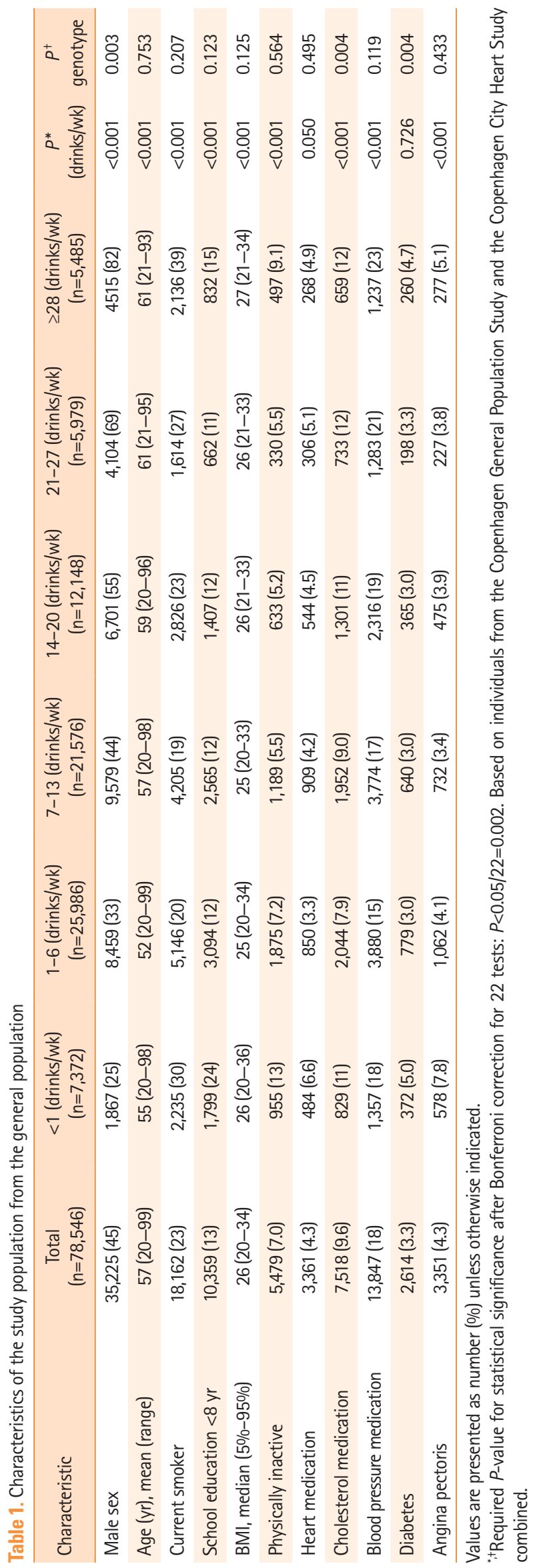

medication (by self-report, yes/no); and use of cholesterollowering medication (by self-report, yes/no). Analyses were conducted using Stata version 12.1 (StataCorp, College Station, TX, USA).

$P$-values for trend between genotypes and baseline characteristics were calculated using chi-square test for categorical variables and Kruskal-Wallis test for continuous variables. For this purpose, genotypes were assigned with values reflecting the effect of genotypes on alcohol consumption.

Participants accrued person-time from baseline until time of stroke $(n=2,335)$, death $(n=7,524)$, emigration $(n=468)$, or end of follow-up $(n=68,161)$, whichever occurred first. Data from the CGPS and CCHS were combined after having ensured that results were similar in the two studies separately. This was tested formally in a nested log-likelihood test $(P=0.16$ for any stroke). Power calculation for the MR analysis were conducted using Stata procedure power logrank. Analyses were conducted following the four steps below, in accordance with the Mendelian randomization study principle. ${ }^{17,40}$

\section{Self-reported alcohol intake and stroke}

We tested whether alcohol intake was associated with stroke using Cox proportional hazards models with delayed entry. Age (in days) was used as the underlying time axis to ensure maximal adjustment for confounding by age. The Cox proportional hazards assumption was examined graphically by plots of log (time) by log (-log [survival probability]) and statistically by introducing interaction terms between age and alcohol intake in the model. No violations were detected ( $P>0.05)$.

\section{Association between $A D H 1 B$ and $A D H 1 C$ genotypes and alcohol intake}

Second, we tested whether $A D H 1 B$ and $A D H 1 C$ genotypes and their combinations were associated with alcohol intake. This was done in linear regression analysis, using the Cuzick's extension of the Wilcoxon rank sum test to calculate $P$-value for trend.

Alcohol and stroke: using ADH1B and ADH1C as instruments for alcohol intake

Third, we tested whether genotype combinations were associated with risk of stroke using Cox regression, modelled as described in 'step 1.'

Alcohol and stroke: observational versus genetic estimates Fourth, to test a potential causal association between alcohol and stroke, we performed instrumental variable analysis with a two-stage regression model using genotype combinations as 


\begin{tabular}{|c|c|c|c|c|c|}
\hline Drinks/week & No. & Events & \multicolumn{2}{|c|}{ Hazard ratio $(95 \% \mathrm{Cl})$} & $P$ for trend,++ \\
\hline \multicolumn{6}{|c|}{ Ischemic stroke } \\
\hline$<1$ & 7,372 & 328 & 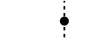 & 1.00 & \\
\hline $1-6$ & 25,986 & 587 & $\bullet$ & $0.86(0.83-0.88)$ & \\
\hline $7-13$ & 21,576 & 521 & 10 & $0.83(0.79-0.88)$ & \\
\hline $14-20$ & 12,148 & 317 & 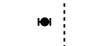 & $0.84(0.79-0.88)$ & \\
\hline $21-27$ & 5,979 & 177 & $\longrightarrow$ & $0.87(0.60-1.28)$ & \\
\hline$\geq 28$ & 5,485 & 202 & $\stackrel{\vdots}{i}$ & $0.98(0.92-1.04)$ & $0.71 /<0.0001$ \\
\hline \multicolumn{6}{|c|}{ Haemorrhagic stroke } \\
\hline$<1$ & 7,372 & 63 & 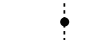 & 1.00 & \\
\hline $1-6$ & 25,986 & 104 & $\longrightarrow \vdots$ & $0.74(0.51-1.08)$ & \\
\hline $7-13$ & 21,576 & 104 & 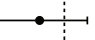 & $0.80(0.52-1.23)$ & \\
\hline $14-20$ & 12,148 & 67 & $\longrightarrow$ & $0.84(0.48-1.49)$ & \\
\hline $21-27$ & 5,979 & 35 & $\rightarrow$ & $0.83(0.50-1.39)$ & \\
\hline$\geq 28$ & 5,485 & 33 & $\rightarrow$ & $0.81(0.29-2.24)$ & $0.75 / 0.51$ \\
\hline \multicolumn{6}{|l|}{ Any stroke } \\
\hline$<1$ & 7,372 & 390 & $\dot{\phi}$ & 1.00 & \\
\hline $1-6$ & 25,986 & 691 & 10-1: & $0.84(0.76-0.92)$ & \\
\hline $7-13$ & 21,576 & 625 & 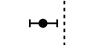 & $0.83(0.73-0.94)$ & \\
\hline $14-20$ & 12,148 & 383 & $\bullet$ & $0.84(0.73-0.97)$ & \\
\hline $21-27$ & 5,979 & 212 & $\longrightarrow$ & $0.87(0.57-1.31)$ & \\
\hline \multirow[t]{2}{*}{$\geq 28$} & 5,485 & 234 & $\mapsto$ & $0.95(0.78-1.17)$ & $0.70 / 0.001$ \\
\hline & & & 1.0 & & \\
\hline
\end{tabular}

Figure 1. Hazard ratio for any stroke, ischemic stroke, and haemorrhagic stroke according to weekly alcohol intake (observational) among men and women $(n=78,546)$. Cl, confidence interval. "Adjusted for sex, smoking status, amount of smoking, school education, body mass index, physical activity, angina pectoris, diabetes, cardiovascular medications, and cohort; ${ }^{\dagger} P$-value for linear trend; ${ }^{\ddagger} P$-value for quadratic trend.

instruments to estimate the effect of per additional genetic drink per day on risk of stroke. ${ }^{16}$ The first stage was a linear regression of genotype combinations on alcohol consumption. Fstatistics $>10$ indicates sufficient statistical strength to carry out statistically valid instrumental variable analysis, which was confirmed $(F=22.0) .{ }^{41}$ The second stage was a logistic regression of alcohol intake determined by genotype combinations (generated in the first stage) on stroke to calculate genetic risk ratios. For comparison, we calculated hazard ratios (HRs) for the association per additional observational drink per day and risk of stroke.

\section{Applicability of included measures}

To test the applicability of the included measures we tested the included measures on already well-established associations, e.g., alcohol intake and risk of alcoholic liver disease and blood pressure and risk of any stroke.

\section{Results}

Baseline characteristics of the 37,344 men and 44,935 women are listed in Table 1, in total and by categories of alcohol intake. Further, $P$-values are shown for associations between each characteristic and alcohol consumption, and between characteristics and $A D H 1 B$ and $A D H 1 C$ genotype combinations. Most characteristics were associated with alcohol consumption. In contrast, none of the baseline characteristics were associated with genotypes after Bonferroni correction for 22 comparisons. This illustrates likely substantial confounding in observational analyses, but none detected in causal, genetic analyses. The mean follow-up duration was 7.9 years. During the study period, 2,535 incident cases of ischemic and haemorrhagic stroke occurred.

Men and women who consumed 1 to 20 drinks/week had reduced risk of any stroke (ischemic and haemorrhagic stroke combined) compared to men and women consuming less than $1 \mathrm{drink} /$ week (Figure 1). No significant association was seen among individuals consuming 21 drinks/week or more. The HRs for any stroke associated with drinking 1 to 6,7 to 13 , and 14 to 20 drinks/week versus less than 1 drink/week were 0.84 (95\% confidence interval [Cl], 0.76 to 0.92$), 0.83(95 \% \mathrm{Cl}, 0.73$ to 0.94$)$, and 0.84 (95\% Cl, 0.73 to 0.97$)$, respectively, and the $P$-value for linear trend was $<0.0001$. The pattern was similar for ischemic and haemorrhagic stroke, but only statistical significant for ischemic stroke.

Evidence of a nonlinear relationship between alcohol intake and total stroke was further supported by Figure 2 showing HRs for any stroke by increasing alcohol intake with $<1$ drink/ 

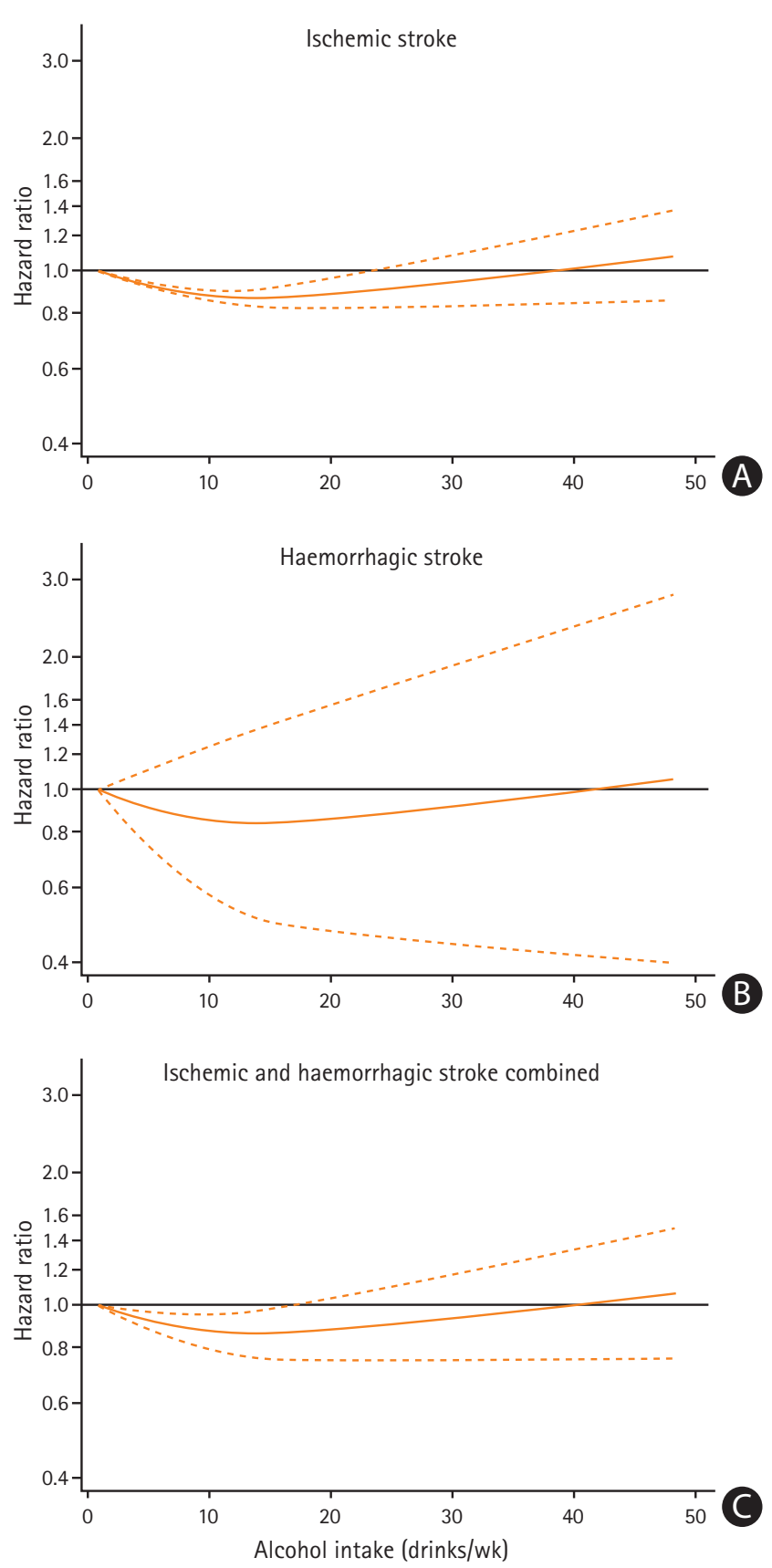

Figure 2. (A-C) Dose-response relationship between alcohol intake and hazard ratios of any stroke (ischemic and haemorrhagic stroke combined), ischemic stroke and haemorrhagic stroke. The solid curve (A) illustrates the hazard ratios and the dashed lines (B) illustrates the 95\% confidence intervals of any stroke, ischemic stroke and haemorrhagic stroke, respectively, by weekly alcohol intake (observational).

week as reference. Indeed, alcohol intake of 1 to 20 drinks/ week was associated with reduced risk of total stroke. Heavy alcohol intake tends to be associated with an increased risk of any stroke, indicating a j-shaped association (Figure 2); however, the association between high alcohol intake and risk of any stroke was not statistically significant (Figure 1).
Further, testing for non-linear trend using the mean alcohol intake for each genotype combination (Table 2), and modelling this amount with a linear and a square term analogous to test for trend as presented in Figure 1 was statically insignificant ( $P=0.40$ and $P=0.42$ for linear and squared terms). Men and women with the $A D H 1 B$ and $A D H 1 C$ slow and intermediate genotypes had higher alcohol consumption than those with the $A D H 1 B$ and $A D H 1 C$ fast genotype (Table 2). For example, individuals with the $A D H 1 B$ and $A D H 1 C$ slow and intermediate genotypes on average drank 22\% and 25\% more alcohol than individuals with the $A D H 1 B$ and $A D H 1 C$ fast genotype. Result from the regression analysis showed that $10 \%$ of the variation in alcohol consumption was explained by the $A D H 1 B$ and $A D H 1 C$ genotypes.

The risk of total stroke was similar among individuals with genotypes coding for high compared with low alcohol consumption: using the $A D H 1 B$ and $A D H 1 C$ genotype combination associated with the lowest alcohol consumption (on average 8.9 drinks/week) as reference, the HR for any stroke for the genotype combination that was associated with the highest alcohol consumption (11.1 drinks/week) was 1.15 (95\% Cl, 0.66 to 2.02) (Table 2). Results obtained in the present study were in keeping with results from the literature, when examining already established association. Hence, increasing alcohol consumption (per 1 drink/day) was positively associated with risk of alcoholic liver disease $(\mathrm{HR}, 1.23 ; 95 \% \mathrm{Cl}, 1.18$ to 1.27$)$, but not associated with risk of any stroke $(\mathrm{HR}, 1.04 ; 95 \% \mathrm{Cl}, 0.98$ to 1.20), and increasing blood pressure (per systolic $10 \mathrm{~mm} \mathrm{Hg}$ ) was not associated with risk of alcoholic liver disease (HR, $1.04 ; 95 \% \mathrm{Cl}, 0.98$ to 1.10 ) but positively associated with risk of any stroke (HR, 1.11; $95 \% \mathrm{Cl}, 1.05$ to 1.17) (Figure 3).

\section{Discussion}

In this prospective cohort study, observational (self-reported) alcohol consumption was associated with lower risk of any stroke (ischemic and haemorrhagic stroke combined) in men and women consuming 1 to 20 drinks/weeks. Heavy alcohol intake if anything tended to be associated with an increase in the risk of any stroke, indicating a j-shaped association. However, no significant association was seen among individuals consuming 21 drinks/week or more. A similar pattern was seen for ischemic and haemorrhagic stroke separately, but this was only significant for ischemic stroke with the most accumulated endpoints. From analyses using genetic variation in alcohol metabolizing genes ( $A D H 1 B$ and $A D H 1 C)$ as instrumental variables for alcohol consumption, we found no evidence to support causality of the observational findings. When examining 
Table 2. Hazard ratio (95\% Cl) for any stroke according to genotype combination in 74,632 men and women

\begin{tabular}{lccrrrr}
\hline ADH1B/ADH1C combination & Relative enzyme activity & No. & Cases & Mean (drinks/wk) & $\Delta(\%)$ & $H R^{*}(95 \% \mathrm{Cl})$ \\
\hline $1(2 / 2+2 / 1 ; 1 / 1)$ & Fastest & 1,701 & 51 & 8.9 & 0 & 1.00 \\
$2(1 / 1 ; 1 / 1)$ & Intermediate (fast) & 24,077 & 750 & 10.8 & 21 & $1.09(0.73-1.62)$ \\
$3(1 / 1 ; 1 / 2)$ & Intermediate (slow) & 35,690 & 1,162 & 10.9 & 22 & $1.13(0.69-1.86)$ \\
$4(1 / 1 ; 2 / 2)$ & Slowest & 13,164 & 429 & 11.1 & 25 & $1.15(0.66-2.02)$ \\
\hline
\end{tabular}

$\mathrm{HR}$, hazard ratio; $\mathrm{Cl}$, confidence interval.

*Adjusted for age and sex.

\begin{tabular}{|c|c|c|c|c|c|c|}
\hline \multirow[b]{2}{*}{ Any stroke } & \multirow{2}{*}{$\begin{array}{r}\text { No. of events } \\
2,535\end{array}$} & \multirow{2}{*}{$\begin{array}{r}\text { Events/1,000 } \\
\text { person-years } \\
40.8\end{array}$} & \multicolumn{2}{|c|}{$\begin{array}{c}\text { Alcohol intake } \\
\text { Hazard ratio }(95 \% \mathrm{Cl})\end{array}$} & \multicolumn{2}{|c|}{$\begin{array}{l}\text { Systolic blood pressure } \\
\text { Hazard ratio }(95 \% \mathrm{Cl})\end{array}$} \\
\hline & & & hor & $1.02(0.99-1.06)$ & $\rightarrow$ & $1.11(1.05-1.17)$ \\
\hline Ischemic stroke & 2,132 & 34.3 & : & $1.03(1.01-1.05)$ & - & $1.11(1.05-1.17)$ \\
\hline Haemorrhagic stroke & 406 & 6.5 & $\longmapsto$ & $1.00(0.92-1.09)$ & $\bullet \bullet$ & $1.09(1.05-1.14)$ \\
\hline Alcoholic liver disease & 150 & 2.4 & \begin{tabular}{c:c} 
& \multicolumn{1}{c}{} \\
0.9 & 1.01 .11 .21 .3 \\
Per 1 drink/day
\end{tabular} & $1.23(1.18-1.27)$ & $\begin{array}{c}c \\
0.91 .01 .11 \\
\text { Per SBP } 10\end{array}$ & $1.04(0.98-1.10)$ \\
\hline
\end{tabular}

Figure 3. Hazard ratios and 95\% confidence intervals ( $\mathrm{Cls}$ ) for any stroke, ischemic stroke, haemorrhagic stroke, and alcoholic liver disease by weekly alcohol intake (observational) and systolic blood pressure (SBP).

already established associations for the included endpoints, the results obtained in the present study are in agreement with results from the literature.

Our results are in accordance with the results in a recent meta-analysis of 20 prospective cohort studies showing that low alcohol intake ( $<15 \mathrm{~g} /$ day, corresponding to less than 1 standard drink), compared with no alcohol intake, was associated with a reduced risk of any stroke ( $\mathrm{RR}, 0.85 ; 95 \% \mathrm{Cl}, 0.75$ to 0.95$)$. Further, compared with no alcohol intake, moderate alcohol intake ( 15 to $30 \mathrm{~g} /$ day, corresponding to approximately 1 to 2 standard drink) showed no significant association with total stroke (RR, $1.01 ; 95 \% \mathrm{Cl}, 0.93$ to 1.09 ) whereas heavy alcohol intake was associated with an increased risk of any stroke. ${ }^{12}$ Low alcohol intake was likewise associated with a reduced risk of ischemic stroke. However, no association between moderate and heavy alcohol consumption and ischemic stroke was evident. Last, the meta-analysis found no association between alcohol intake at any level and risk of haemorrhagic stroke. Another meta-analysis of 27 prospective cohort studies found that light to moderate alcohol consumption ( $\leq 2$ drinks/day) was associated with a reduced risk of ischemic stroke but not associated with haemorrhagic stroke subtypes. ${ }^{13}$ Further, heavy drinking ( $>4$ drinks/day) was found to be associated with all stroke types.

\section{Plausible explanations}

Our result and the dose-response meta-analysis suggest that a potential nonlinear relationship is present between alcohol intake and risk of any stroke, and that an alcohol intake of 1 to 20 drinks/week is associated with a reduced risk of any stroke.
Hence, the association seems to be j-shaped. The divergent dose-response relationships between alcohol consumption and risk of ischemic and haemorrhagic stroke subtypes suggest that different mechanisms underlie associations with the different stroke types. Alternatively, the slightly different results may be due to fewer haemorrhagic stroke events compared with ischemic stroke events, and thus the statistical power could be too low for reliable risk estimates for haemorrhagic stroke alone. Heavy alcohol intake may be associated with an increased risk of haemorrhagic events because heavy alcohol intake potentially results in high blood pressure, reduced platelet aggregation, and enhanced fibrinolysis. ${ }^{12,13}$ Further, alcohol consumption is associated with increased high density lipoprotein cholesterol levels, decrease platelet aggregation, increase fibrinolysis, and decrease plasma fibrinogen levels and this might help explain the lower risk of ischemic stroke. ${ }^{12,13}$

\section{Strengths and limitations}

From analysis using $A D H$ genotypes as instrument for alcohol consumption, we did not obtain results that supported causality of the observational finding. The strength of using genetic instruments for lifestyle exposures is that confounding from associated risk factors and reverse causation are unlikely, and causal interpretation of results may be more straight forward than causal interpretation of observational findings. ${ }^{17}$ Further, selection bias due to non-participation is less likely to affect results for genetic instruments ${ }^{42}$ whereas as for results obtained for self-reported alcohol intake, bias cannot be ruled out: if alcohol intake was associated with non-participation 
and with risk of stroke, results would be influenced. ${ }^{43}$ Hence, heavy drinkers who participate may be at better health than heavy drinkers in the underlying population, whereas light and moderate drinkers who volunteer may be more representative of moderate and non-drinkers drinkers generally. Ultimately, such a mechanism would lead to more accurate results among the light to moderate drinkers and underestimated results among the heavy drinkers.

A limitation is that the $A D H 1 B$ and $A D H 1 C$ genotypes are not strong instruments, meaning that the influence on the amount of alcohol consumption is limited; $10 \%$ of the variation in alcohol intake was explained by the $A D H 1 B$ and $A D H 1 C$ genotypes in this population. Hence, the lack of a positive finding does not preclude that alcohol is causally associated with stroke. Further, using these genotypes as instruments implies a linear model between genetic alcohol consumption and the risk of stroke; if the association between alcohol and risk of stroke for instance is indeed threshold-shaped, then the genetic instrument has little power to pick it up.

Further, another limitation is lack of power in the MR analysis. We computed the lowest HR that can be picked up with the present set-up, for the ADH1B/ADH1C combination with the highest alcohol intake (i.e., the 1/1;1/2 group, please refer to Table 2), with a 90\% power and a significance level of 0.05 : this $H R$ is 0.65 , meaning that we do not have the adequate power to assess a HR of 0.84 .

The reason that $A D H$ genotypes is associated with alcohol consumption is most likely that-for a given level of intake-individuals with fast alcohol degradation have higher levels of acetaldehyde leading to unpleasant symptoms such as nausea and flushing compared with individuals with slow degradation, causing them to drink less. Thus, peak levels of acetaldehyde, known to be carcinogenic and playing important roles in the development of alcohol-related diseases, ${ }^{44}$ will be higher in fast compared to slow metabolisers, meaning that by using $A D H 1 B$ and $A D H 1 C$ genotypes as instruments the difference in amount of alcohol cannot be studied independently of difference in acetaldehyde levels, which constitute a final limitation of this approach.

A candidate for another instrument of alcohol intake to be used in future MR studies is the recently identified single nucleotide polymorphism (SNP) in Klotho $\beta$ (KLB) gene (encoding $\beta$-Klotho), demonstrated to have a role in the regulation of alcohol drinking, observed in independent samples. ${ }^{45}$ While the mechanism linking the KLB gene with alcohol intake is still unknown, making the potential for using it as an instrument for alcohol intake unsure it is a promising candidate to be used alone or in combination with $A D H 1 B$ and $A D H 1 C$ genotypes forming stronger instruments. Last, it was not possible to separate former drinkers from abstainers and hence, some drinker misclassification might have occurred.

Study strengths include the large number of individuals and stroke endpoints, and that the hospital register information on the stroke diagnosis was highly sensitive and specific. ${ }^{36,37}$ Further, the National Danish Patient Register covers all hospitalizations in Denmark including outpatients and emergency wards since 1995, and only if participants were treated in another country or by a general practitioner solely, the information would be lacking. Hence, loss to endpoint findings is considered to be negligible.

\section{Conclusions}

In this pooled analysis of 78,546 individuals, self-reported alcohol intake of 1 to 20 drinks/week was associated with reduced risk of any stroke. The risk of any stroke if anything tended to be increased among individuals with heavy alcohol intake, indicating a j-shaped association. However, no significant association was found between high alcohol consumption and risk of any stroke. The pattern was similar for ischemic stroke and haemorrhagic stroke, but only statistically significant for ischemic stroke. Finally, we found no evidence to support a causal relation of a linear association between alcohol intake and total stroke from genetic estimates. However, this could be explained by lack of power in the MR analysis.

\section{Disclosure}

The authors have no financial conflicts of interest.

\section{Acknowledgments}

We thank the participants of the Copenhagen City Heart Study and the Copenhagen General Population Study for their time and cooperation. The study was funded by The European Foundation for Alcohol Research.

\section{References}

1. Stampfer MJ, Colditz GA, Willett WC, Speizer FE, Hennekens $\mathrm{CH}$. A prospective study of moderate alcohol consumption and the risk of coronary disease and stroke in women. N Engl J Med 1988;319:267-273.

2. Lu M, Ye W, Adami HO, Weiderpass E. Stroke incidence in women under 60 years of age related to alcohol intake and smoking habit. Cerebrovasc Dis 2008;25:517-525. 
3. Bos S, Grobbee DE, Boer JM, Verschuren WM, Beulens JW. Alcohol consumption and risk of cardiovascular disease among hypertensive women. Eur J Cardiovasc Prev Rehabil 2010;17:119-126.

4. Klatsky AL, Armstrong MA, Friedman GD. Alcohol use and subsequent cerebrovascular disease hospitalizations. Stroke 1989;20:741-746.

5. Mukamal KJ, Chung H, Jenny NS, Kuller LH, Longstreth WT Jr, Mittleman MA, et al. Alcohol use and risk of ischemic stroke among older adults: the cardiovascular health study. Stroke 2005;36:1830-1834.

6. Donahue RP, Abbott RD, Reed DM, Yano K. Alcohol and hemorrhagic stroke. The Honolulu Heart Program. JAMA 1986;255: 2311-2314.

7. Iso $H$, Kitamura $A$, Shimamoto $T$, Sankai $T$, Naito $Y$, Sato $S$, et al. Alcohol intake and the risk of cardiovascular disease in middle-aged Japanese men. Stroke 1995;26:767-773.

8. Leppälä JM, Paunio M, Virtamo J, Fogelholm R, Albanes D, Taylor PR, et al. Alcohol consumption and stroke incidence in male smokers. Circulation 1999;100:1209-1214.

9. Djoussé L, Ellison RC, Beiser A, Scaramucci A, D'Agostino RB, Wolf PA. Alcohol consumption and risk of ischemic stroke: the Framingham Study. Stroke 2002;33:907-912.

10. Nielsen NR, Truelsen T, Barefoot JC, Johnsen SP, Overvad K, Boysen $\mathrm{G}$, et al. Is the effect of alcohol on risk of stroke confined to highly stressed persons? Neuroepidemiology 2005;25:105-113.

11. Bazzano LA, Gu D, Reynolds K, Wu X, Chen CS, Duan X, et al. Alcohol consumption and risk for stroke among Chinese men. Ann Neurol 2007;62:569-578.

12. Zhang $C$, Qin $Y Y$, Chen $Q$, Jiang $H$, Chen $X Z, X u C L$, et al. Alcohol intake and risk of stroke: a dose-response meta-analysis of prospective studies. Int J Cardiol 2014;174:669-677.

13. Larsson SC, Wallin A, Wolk A, Markus HS. Differing association of alcohol consumption with different stroke types: a systematic review and meta-analysis. BMC Med 2016;14:178.

14. Ng Fat $L$, Cable N, Marmot MG, Shelton N. Persistent longstanding illness and non-drinking over time, implications for the use of lifetime abstainers as a control group. J Epidemiol Community Health 2014;68:71-77.

15. Choi NG, DiNitto DM, Marti CN, Choi BY. Sociodemographic characteristics and health status of lifetime abstainers, exdrinkers, bingers, and nonbingers among baby boomers and older adults. Subst Use Misuse 2016;51:637-648.

16. Palmer TM, Sterne JA, Harbord RM, Lawlor DA, Sheehan NA, Meng $S$, et al. Instrumental variable estimation of causal risk ratios and causal odds ratios in Mendelian randomization analyses. Am J Epidemio/ 2011;173:1392-1403.

17. Smith GD, Ebrahim S. 'Mendelian randomization': can genet- ic epidemiology contribute to understanding environmental determinants of disease? Int J Epidemio/ 2003;32:1-22.

18. Tolstrup JS, Grønbaek M, Tybjaerg-Hansen A, Nordestgaard $B G$. Alcohol intake, alcohol dehydrogenase genotypes, and liver damage and disease in the Danish general population. Am J Gastroenterol 2009;104:2182-2188.

19. Lawlor DA, Nordestgaard BG, Benn M, Zuccolo L, TybjaergHansen A, Davey Smith G. Exploring causal associations between alcohol and coronary heart disease risk factors: findings from a Mendelian randomization study in the Copenhagen General Population Study. Eur Heart J 2013;34:2519-2528.

20. Kumari M, Holmes MV, Dale CE, Hubacek JA, Palmer TM, Pikhart $\mathrm{H}$, et al. Alcohol consumption and cognitive performance: a Mendelian randomization study. Addiction 2014;109:14621471.

21. Muñoz $X$, Amiano $P$, Celorrio $D$, Dorronsoro $M$, Sánchez MJ, Huerta JM, et al. Association of alcohol dehydrogenase polymorphisms and life-style factors with excessive alcohol intake within the Spanish population (EPIC-Spain). Addiction 2012;107:2117-2127.

22. Way M, McQuillin A, Saini J, Ruparelia K, Lydall GJ, Guerrini I, et al. Genetic variants in or near ADH1B and ADH1C affect susceptibility to alcohol dependence in a British and Irish population. Addict Biol 2015;20:594-604.

23. Wium-Andersen MK, Orsted DD, Nordestgaard BG. Elevated C-reactive protein, depression, somatic diseases, and allcause mortality: a mendelian randomization study. Biol Psychiatry 2014;76:249-257.

24. Wium-Andersen MK, Ørsted DD, Tolstrup JS, Nordestgaard BG. Increased alcohol consumption as a cause of alcoholism, without similar evidence for depression: a Mendelian randomization study. Int J Epidemiol 2015;44:526-539.

25. Bjerregaard P, Mikkelsen SS, Becker U, Hansen T, Tolstrup JS. Genetic variation in alcohol metabolizing enzymes among Inuit and its relation to drinking patterns. Drug Alcohol Depend 2014;144:239-244.

26. Pedersen CB. The Danish civil registration system. Scand J Public Health 2011;39(7 Suppl):22-25.

27. Schnohr $P$, Jensen $G B$, Appleyard $M$, Lange $P$, Nordestgaard BG, Grønbæk M, et al. The Copenhagen City Heart Study: østerbroundersøgelsen. Tables with data from the third examination 1991-94. Eur Heart J Supp/ 2001;3(Suppl H):H1-H83.

28. Thomsen $M$, Ingebrigtsen TS, Marott JL, Dahl M, Lange $P$, Vestbo $J$, et al. Inflammatory biomarkers and exacerbations in chronic obstructive pulmonary disease. JAMA 2013;309:2353-2361.

29. Jørgensen $A B$, Frikke-Schmidt $R$, Nordestgaard BG, TybjærgHansen A. Loss-of-function mutations in APOC3 and risk of ischemic vascular disease. N Engl J Med 2014;371:32-41. 
30. Mukamal KJ, Tolstrup JS, Friberg J, Jensen G, Grønbaek M. Alcohol consumption and risk of atrial fibrillation in men and women: the Copenhagen City Heart Study. Circulation 2005; 112:1736-1742.

31. Sethi AA, Tybjaerg-Hansen A, Andersen RV, Nordestgaard BG. Nanogen microelectronic chip for large-scale genotyping. Clin Chem 2004;50:443-446.

32. Tolstrup JS, Nordestgaard BG, Rasmussen S, Tybjaerg-Hansen A, Grønbaek M. Alcoholism and alcohol drinking habits predicted from alcohol dehydrogenase genes. Pharmacogenomics J 2008;8:220-227.

33. Andersen TF, Madsen $M$, Jørgensen J, Mellemkjoer $L$, Olsen JH. The Danish National Hospital Register. A valuable source of data for modern health sciences. Dan Med Bull 1999;46:263268.

34. Helweg-Larsen K. The Danish register of causes of death. Scand J Public Health 2011;39(7 Suppl):26-29.

35. Juel K, Helweg-Larsen K. The Danish registers of causes of death. Dan Med Bull 1999;46:354-357.

36. Truelsen T, Grønbaek M, Schnohr P, Boysen G. Stroke case fatality in Denmark from 1977 to 1992: the Copenhagen City Heart Study. Neuroepidemiology 2002;21:22-27.

37. Ellervik C, Tybjaerg-Hansen A, Appleyard M, Sillesen H, Boysen G, Nordestgaard BG. Hereditary hemochromatosis genotypes and risk of ischemic stroke. Neurology 2007;68:1025-1031.
38. Hatano S. Experience from a multicentre stroke register: a preliminary report. Bull World Health Organ 1976;54:541-553.

39. Brøndum-Jacobsen P, Nordestgaard BG, Schnohr P, Benn M. 25-Hydroxyvitamin D and symptomatic ischemic stroke: an original study and meta-analysis. Ann Neurol 2013;73:38-47.

40. Nordestgaard BG, Palmer TM, Benn M, Zacho J, Tybjaerg-Hansen A, Davey Smith G, et al. The effect of elevated body mass index on ischemic heart disease risk: causal estimates from a Mendelian randomisation approach. PLoS Med 2012;9:e1001212.

41. Lawlor DA, Harbord RM, Sterne JA, Timpson N, Davey Smith G. Mendelian randomization: using genes as instruments for making causal inferences in epidemiology. Stat Med 2008;27:11331163.

42. Smith GD, Ebrahim S. Mendelian randomization: prospects, potentials, and limitations. Int J Epidemiol 2004;33:30-42.

43. Hernán MA, Hernández-Díaz $S$, Robins JM. A structural approach to selection bias. Epidemiology 2004;15:615-625.

44. Guo R, Ren J. Alcohol and acetaldehyde in public health: from marvel to menace. Int J Environ Res Public Health 2010;7: 1285-1301.

45. Schumann G, Liu C, O'Reilly $P$, Gao H, Song P, Xu B, et al. KLB is associated with alcohol drinking, and its gene product beta-Klotho is necessary for FGF21 regulation of alcohol preference. Proc Natl Acad Sci U S A 2016;113:14372-14377. 